\title{
Combining two approaches to efficiency assessment
}

\author{
Dr. Chris Tofallis \\ Dept. of Statistics, Economics, Accounting and Management Systems \\ University of Hertfordshire Business School \\ Mangrove Road \\ Hertford, SG13 8QF \\ U.K.
e-mail: c.tofallis@ herts.ac.uk tel: +44 (0) 1707285486 fax: +44 (0) 1707285489

Keywords: data envelopment analysis, econometrics, performance measurement, regression, statistics.

\begin{abstract}
$\underline{\text { Abstract }}$
The advent of data envelopment analysis (DEA) enabled the measurement of efficiency to be extended to the case of multiple outputs. Prior to DEA we had the parametric approach based on multiple regression. We highlight some difficulties associated with these two approaches and present a hybrid which overcomes them whilst maintaining the respective advantages of each. This hybrid models the efficient frontier using an algebraic expression; the resulting smooth representation allows all units to be naturally enveloped and hence slacks to be avoided. (Slacks are potential improvements for inefficient units which are not accounted for in the DEA (radial) score, and so have been problematic for DEA.)

The approach identifies the DEA-efficient units and fits a smooth model to them using maximum correlation modelling. This new technique extends the method of multiple regression to the case where there are multiple variables on each side of the model equation (e.g. outputs and inputs). The resulting expression for the frontier permits managers to estimate the effect on their efficiency score of adjustments in one or more input or output levels.
\end{abstract}




\section{Introduction}

Given a set of decision making units (DMUs: departments, branches, firms etc.) to be compared there are two main approaches for the construction of an efficient frontier upon which efficiency scores can be based. One is the parametric approach, often used by economists, the other is the non-parametric approach known as data envelopment analysis (DEA). We shall outline these and point out their drawbacks. The purpose of this paper is to propose a hybrid approach which not only brings together the useful aspects of these two methods but also avoids their limitations.

In both of the standard approaches mentioned above the efficiency of an organisational unit is measured by reference to a frontier. There are two types of parametric frontier: deterministic and stochastic. In the former a given functional form which relates output to the various inputs (e.g. a Cobb-Douglas function) is fitted using regression constrained so that all points lie on one side of the (production) frontier. This is achieved by restricting the residuals to be one-sided i.e. all of the same sign. This means that no unit can produce more output than that calculated from this frontier production function for its given levels of inputs. With stochastic frontiers we have both unrestricted residuals as well as one-sided ones within the same model. The idea being that the former will represent random effects such as the weather and equipment failures, while the latter indicate inefficiency. Unfortunately a specific distribution must be assumed for the residuals before the model can be deduced; this implies that we must know a priori what the distribution of inefficiency is.

One problem with parametric methods is that we have no indication of how well the function fits to the frontier units, this is important because the function is meant to model these efficient units only. The problem arises because least squares regression minimises the sum of squared residuals for all units - the inefficient as well as the efficient. Hence the $\mathrm{r}^{2}$ value is not appropriate for our purpose. The effect of a mis-specified functional form will be confounded with inefficiency. What is evidently required is to first identify the frontier units and then select a function according to how well it fits to these units alone. This is the direction that we shall take.

Secondly, in almost all published cases where the parametric approach is used there is only a single output and this quantity, or its logarithm, is the dependent variable in the frontier production function that is fitted. According to Greene': 'The frontiers literature contains results for multiple output technologies, though in these cases it is necessary to work with 
cost, revenue and profit functions'. Of course there are many areas of performance measurement where it is very difficult, if not impossible, to attach monetary values to the various criteria, education and health being two examples. DEA provides a way of dealing with multiple outputs which avoids attributing monetary values to them.

The non-parametric approach DEA is specifically designed to deal with multiple outputs and inputs. By solving a set of linear programmes, one for each DMU, it identifies the frontier units and constructs a piece-wise linear frontier which passes through each of them. This means that, unlike the parametric method, we do not have a single functional form to represent the efficient frontier. Having such a formula would provide a simple and convenient way to see the effect of changes in inputs and/or outputs on the efficiency score for any given production unit. A formula is also useful for establishing targets for particular inputs or outputs. Simar' ${ }^{2}$ points out that 'a parametric form for the production function allows for a richer economic interpretation of the production process'.

In DEA a point not on the frontier is termed 'inefficient' and its efficiency score is based on a comparison with some point which does lie on the frontier, usually one with a similar mix of inputs or outputs. The most common way of selecting such a 'reference point' is by a radial projection from the origin which passes through the point being assessed and then intersects the frontier. Such a reference point represents a linear combination of other efficient units. In Figure 1 the points A, B and D represent efficient production units, whereas points $\mathrm{P}$ and $\mathrm{C}$ are inefficient because they lie behind the frontier constructed from the efficient units. Thus in Figure 1 the efficiency of unit $\mathrm{P}$ is calculated as the ratio OP/OP'. A serious difficulty arises when the radial projection does not encounter the frontier; this occurs with unit $\mathrm{C}$. Geometrically speaking the frontier in output space does not reach the co-ordinate planes, so that there is an 'uncovered area' around it. Hence some units are not 'naturally enveloped' because the frontier is incomplete. For this problem not to arise would require the presence of efficient units each of which has a zero value for one of the outputs, the frontier would then reach all the way to the co-ordinate planes and no rays from the origin could 'escape' around it. Such data sets almost never arise in reality.

DEA deals with this problem by artificially extending the frontier parallel to the coordinate planes (dashed line in Figure 1). The points along this extension will not be efficient because moving along such an extension implies that one of the inputs or outputs must be changing with the other variables remaining at the same level. In other words any point on the extension is dominated by a point on the edge of the efficient frontier. In our example point $\mathrm{C}^{\prime}$ is dominated by $\mathrm{D}$. The extent of this dominance is measured by the difference in input or 
output between these two points and this is called 'slack'. Hence in DEA the potential for improvement for an inefficient unit is made up of two parts, the radial (i.e. equiproportionate) expansion of outputs, or contraction of inputs, and the adjustments arising from slacks, which are not equiproportionate. As DEA efficiency scores do not take account of the slacks they are an incomplete measure, and it is necessary to list these slacks separately for each of the inputs and outputs. Sadly this is not often done in the literature and one is left with a league table of misleading scores. Consider two inefficient units with the same score, if one has slack and the other does not, then the latter is clearly more efficient and should be ranked higher, but it is unclear by how many positions. If instead both these units have slacks but in different outputs/inputs then it is unclear how to deal with ranking. Therefore DEA scores should not be used for ranking the units, although they often are. An attempt at avoiding slacks by extending the frontier in a different way (Bessent et $\mathrm{al}^{3}$ ) was only partially successful in that many units were still not naturally enveloped. Torgersen et $\mathrm{al}^{4}$ found that the potential for output increase contained in the slacks can often exceed that indicated by the radial efficiency score. They proposed that a unit's efficiency in each dimension be reported, so for output efficiency one would use the ratio (actual output)/(total potential output), where the denominator is the potential output due to radial expansion summed with the slack in that output. Lovell and Eeckaut ${ }^{5}$ propose a non-radial measure and point out that "the slack component is widely ignored in DEA analyses of producer performance. This practice leads to an overstatement of technical efficiency, it distorts rankings of producers on the technical efficiency criterion, and it suppresses useful information". Another aspect of the artificial extension of the frontier beyond what is empirically observed is that an unenveloped unit (such as C) will be given a target that involves a significant change in the output mix (D) after the slack adjustment has been made. Hartman and Storbeck ${ }^{6}$ encountered this problem when assessing a food manufacturing organisation, the targets for some units were felt to be too different to be useful.

There are also problems with DEA weights. Firstly, the weights or 'multipliers' attached to the outputs can be used to calculate trade-offs between them, and input weights allow input substitution rates to be found. Along a particular DEA frontier facet these transformation rates will have a constant value but they then change suddenly and discontinuously when an adjacent facet is encountered. In reality these rates would be expected to change smoothly - if we were fortunate enough to have an unlimited quantity of data points we would expect the frontier to appear as a smooth surface. Secondly, frontier units in DEA occur at the intersection of two or more facets and so the weights that such units 
employ are not unique: one can use the weights associated with any of the intersecting facets as well as an infinity of intermediate combinations.

In summary the parametric approach provides a convenient formula relating output to inputs but is not based on a clearly identified set of efficient units. Also it is not simply extendible to multiple outputs. In contrast, DEA has the advantages of clearly identifying the efficient units and easily incorporating multiple outputs, but it does not provide a single formula to model the efficient frontier and it suffers from the problems associated with slacks. In the next section we present an approach which not only retains the advantages of both methods but also avoids all the drawbacks we have mentioned.

\section{A hybrid approach}

Since DEA is well suited for identifying the frontier production units, we first apply this technique to the data. In the second stage we set aside the non-frontier (inefficient) units and fit a smooth model to the efficient (frontier) units only. We can attempt a number of models until we are satisfied that we have achieved a good fit. We can now legitimately use $\mathrm{r}^{2}$ or any other measure of goodness of fit to assess how well we have modelled the efficient units, since unlike the parametric methods of econometrics we are no longer including the non-frontier units. We also show how to fit a model incorporating multiple outputs something not directly possible using standard regression.

The idea of filtering out inefficient units before fitting a parametric model has been used by Thiry and Tulkens ${ }^{7}$ and also Simar ${ }^{2}$. They refer to this as a semi-parametric approach. Although they did not use DEA for screening out the inefficient units, they did point out that possibility. Instead they applied a free disposal hull (FDH) method. Their work uses regression to fit the frontier model and so is restricted to a single output as dependent variable.

We now describe how we can go beyond multiple regression to construct models involving both multiple outputs and multiple inputs. Canonical correlation analysis (CCA) is a technique for finding linear combinations of two sets of variables such that the correlation between these quantities ('canonical variates') is maximised. It is available in some of the more comprehensive statistical software packages, although as we shall see, they will not be required for our purposes because of their limitations. Applying CCA to our case we might start by seeking a linear combination $(\mathrm{Y})$ of outputs and another $(\mathrm{X})$ for the inputs. A high 
correlation between $\mathrm{X}$ and $\mathrm{Y}$ would imply that regression between these two composite quantities would provide a model relating multiple outputs and inputs.

The idea of applying CCA to the DEA-efficient set appeared as a suggestion at the end of a paper by Sengupta ${ }^{8}$. Canonical correlation has since been used by Friedman and Sinuany-Stern ${ }^{9}$, but on the full set of DMUs, i.e. they were not trying to model the efficient frontier. Their purpose was to generate a common set of weights for the purpose of ranking units on a common scale. They pointed out that negative weights could arise and would be problematic. Their advice for dealing with this was that one should first check if an input had been mis-classified as an output or vice versa. If this were not the case they suggested simply removing the offending variable. Arnold et $\mathrm{al}^{10}$ did restrict their canonical correlation analysis to the DEA-efficient subset. They used the logarithms of the inputs and outputs and their purpose was to generate an aggregate output for subsequent use in a stochastic frontier determination, the canonical input variate was not used. They did encounter negative coefficients/weights but chose to retain them.

In this paper we avoid negative coefficients (and also permit the inclusion of other constraints) by discarding the standard computational procedure used for CCA, which involves solving an eigenvector problem where the largest eigenvalue corresponds to the square of the maximum correlation between canonical variates. ${ }^{11}$ Instead we shall treat the problem as a constrained optimisation: maximise the correlation between a function of the inputs $X=f(x)$ and a function of the outputs $Y=g(y)$ subject to a set of constraints . The constraints need not merely be non-negativities, they can represent any prior knowledge, intuitively obvious requirements or theoretical features that the model should possess e.g. to ensure convexity or to avoid the possibility of congestion (backward bending of the frontier) which may arise with some types of function. We thus have much greater control of the final model. Since, in general, the resulting model will differ from that arising from CCA, we might distinguish the two by referring to ours as a constrained CCA model (CCCA) or, more briefly and descriptively, as a maximum correlation model.

We can show that the resulting solution to our constrained optimisation will be a global optimum. If the functions of outputs and inputs, $\mathrm{Y}$ and $\mathrm{X}$, are linear in their coefficients and if the constraints also have this property, then we have a quadratic programming problem. Since we shall be dealing with positive values of inputs and outputs we shall have a positive definite quadratic form in the objective function. Maximising this over our convex feasible region guarantees that the optimum we find will be global. 
It must be emphasised that we can have non-linear functions of the inputs and outputs in our model as well as interaction terms (i.e. cross-products such as $\mathrm{x}_{1} \mathrm{x}_{2}$ ) whilst still retaining linearity in their coefficients. Moreover all such models can now be derived using spreadsheet software which now contain optimisation solvers as a standard tool. All the models in this paper were estimated using Microsoft Excel, but could equally have been generated in Lotus 123, or Quattro Pro.

In summary, our approach consists of three steps:

I. Apply DEA to identify the efficient units.

II. Using these efficient units only, maximise the correlation between a selected function $(\mathrm{X})$ of the inputs and a function (Y) of the outputs, these functions will contain coefficients or weights whose values will be found subject to constraints which force the model to possess certain properties. These conditions may, for instance, be chosen to permit easy interpretation. A correlation value leads one to proceed to step III, otherwise one attempts a different model by changing the selected functions to achieve a better fit.

III. A high correlation implies that a simple regression can now be performed between $\mathrm{Y}$ and $\mathrm{X}$ that will provide a model that fits the efficient units well. We will then have a smooth function to represent the frontier.

\section{An illustrative application}

The data set we shall use deals with chemistry departments in 52 British universities and has been extracted from Beasley ${ }^{12}$ or 13 . It can be downloaded from the collection of data sets known as the OR Library at http://mscmga.ms.ic.ac.uk/info.html. We use two inputs: general expenditure (GEN) - mainly staff salaries, and equipment expenditure (EQU), both measured in thousands of pounds sterling. The four outputs we shall use are the number of undergraduates (UG), number of taught postgraduates (PGT), the number of research postgraduates (PGR), and research income (RSCH) in thousands of pounds. We stress from the outset that this is not intended as a serious comparison of these departments; this would require data spanning several years since equipment expenditure tends to vary widely from one year to the next. There may also be some disagreement regarding the choice of variables and whether the research income that a department attracts should be treated as an output (as here) or an input (as in Beasley). With this is mind we proceed with what is after all only an illustrative application. 
Applying the Banker, Charnes, Cooper DEA output maximisation model, which assumes variable returns to scale, we find 22 efficient departments. In fact the same units were efficient in the input minimisation model. (It should be noted that if constant returns to scale are assumed then, in general, there will be fewer efficient units arising because the fit to the data is not as tight, this in turn leads to having less data with which to model efficient practice.) We next attempt to find a function of the outputs and a function of the inputs so that they have a high correlation for this efficient subset of the data. We first try simple linear combinations of the inputs and outputs $(\mathrm{X}=\Sigma a x, \mathrm{Y}=\Sigma$ by). Hence our optimisation problem is to find coefficient values $\left(a_{1}, a_{2}, b_{1}, b_{2}, b_{3}, b_{4}\right)$ which maximise the correlation between the linear combinations ( $\mathrm{X}$ and $\mathrm{Y}$ ) subject to the coefficient values being non-negative. (See the Appendix for the mathematical details.) This leads to the following solution where we have scaled each of these expressions so as to arrange for one of the coefficients to equal unity:

$$
\begin{aligned}
& \mathrm{X}=\mathrm{GEN}+0.667 \mathrm{EQU} \\
& \mathrm{Y}=0.04 \mathrm{RSCH}+\mathrm{UG}+2.43 \mathrm{PGT}+4.38 \mathrm{PGR}
\end{aligned}
$$

When this problem was originally solved using a canonical correlation analysis routine in a commercial statistical software package it returned some negative coefficients and this is what prompted the current research. Subsequent testing of the routine and comparison with results from other statistical software showed it to be incorrect. It so happens that in this instance removal of the non-negativity conditions leads to the same model, thus this avenue of research was initiated as a result of an accident. Nevertheless there is a clear need for the extension to the method that we describe. In concluding what is perhaps the most comprehensive monograph on CCA, Gittins ${ }^{14}$ says "Canonical analysis is known and appreciated more for its elegant mathematical properties than for its utility. Convincing practical examples specifically of CCA remain comparatively few". The problem is that CCA results are not always easily interpretable and this is blamed for its relative scarcity in the research literature ${ }^{15}$. In general we shall find a reduced value of the correlation once constraints are applied. Therefore there is usually a trade-off between the goodness of fit of the model and its acceptability on theoretical or intuitive grounds. One expects the preference to be in favour of making some sacrifice in goodness of fit in order to have a model that is meaningful and can be interpreted. 
In our case the correlation between $\mathrm{X}$ and $\mathrm{Y}$ was 0.976. As this is a high value we proceed to construct a regression model. This gives the following expression for the expected efficient output value (denoted by an italic symbol $Y$ ) for given inputs:

$$
Y=0.4133 \mathrm{X}+5.2 \quad\left(\mathrm{r}^{2}=0.953\right)
$$

(N.b. the value of the constant term is negligible in comparison to the magnitude of the other term.)

From this we can calculate the output efficiency as $Y / Y$ i.e. the ratio of the actual output function to the efficient output function. Note that any ranking based on this ratio will be identical to that based on $\mathrm{Y} / \mathrm{X}$ because $Y$ is linearly related to $\mathrm{X}$. One convenience arising from the regression that gives rise to $Y$ is that the value of the ratio $\mathrm{Y} / Y$ will roughly lie in the range zero to one (some units will have scores exceeding unity as a result of imperfect fit to the frontier).

In order to illustrate construction of a nonlinear (curved) function of the variables let us instead suppose that we were not satisfied with the above fit. Before attempting to do this we choose to remove the outlier (Oxford) because a scatter-plot shows this is a point possessing high leverage i.e. it would be very influential in forcing us to retain an almost linear fit. (Whether the removal of an outlier is valid from an efficiency assessment point of view is debatable, but we are merely doing this for the purpose of illustration). We now find that if we take logs of the inputs and proceed as before to find the maximum correlation model we obtain an improved correlation of 0.988 with

$$
\begin{aligned}
& \mathrm{X}=\ln (\mathrm{GEN})+0.1036 \ln (\mathrm{EQU}) \\
& \mathrm{Y}=\mathrm{RSCH}+23.63 \mathrm{UG}+80.3 \mathrm{PGT}+70.4 \mathrm{PGR}
\end{aligned}
$$

(Logs were not used for the outputs as these occur at zero values for some units.)

The regression equation is: $\quad Y=7204 \mathrm{X}-42973 \quad\left(\mathrm{r}^{2}=0.977\right)$

If we now use this to calculate the output efficiency $\mathrm{Y} / Y$ (i.e. the ratio of the actual output index to the expected efficient output index) we find that the subset of 21 DEA-efficient units used in the regression have scores with the following properties: all but two units have scores in the range $90 \%$ and $107 \%$ (our regression provides an average formula for the efficient units, the fact that some will score above $100 \%$ can be interpreted as due to external forces contributing random noise or alternatively this effect can be avoided by a downshift of the frontier). The lowest score among the DEA-efficient units is $84 \%$ and the unit concerned is 
one which was not referenced by any other inefficient units as a comparator (reference point) in the DEA investigation i.e. its apparent efficiency rested on the absence of other units with which a comparison could be made - such units are sometimes referred to as mavericks and usually appear efficient in DEA as a result of unusual/extreme weights being attached to the inputs or outputs. It is perhaps pleasing to note that our approach picked up and downgraded this unit. Charnes et $\mathrm{al}^{16}$ cite a DEA investigation in which a few notoriously inefficient banks were classed as efficient; they resorted to imposing restrictions on the DEA weights to obtain more realistic scores. DEA without weight restrictions permits extreme weight values and this corresponds to a frontier with large discontinuous changes in the direction of the frontier as we move from one linear facet to the next in order to include a maverick unit. There is a body of literature in DEA devoted to weight restrictions with the purpose of obtaining more practically acceptable weights (see Allen et $\mathrm{al}^{17}$ or Dyson and Thanassoulis ${ }^{18}$ ). However choosing such upper and lower limits is not easy; we have managed to avoid doing this by employing a smooth frontier. (An alternative way of dealing with mavericks is to use crossefficiency ${ }^{19}$, this is grounded in the notion of aggressive or benevolent peer appraisal.)

The effect of this new frontier on the DEA-inefficient units is interesting because now the scores cannot hide inefficiencies due to slacks. Every single one of the thirty inefficient units had slack in at least one output i.e. there were additional output gains over and above those implied by the DEA score. As a result we expect, and do actually find, changes in the rankings of these units (see Table 1, where the ranks are based on the inefficient subset alone). Departments with large slacks in a number of outputs should fall in the rankings, whereas those with a small slack in only one output should rise. By dividing the actual output by the target output from DEA (including slack), we can obtain an efficiency score for each output; from these four figures DEA chooses the largest as the final score, any that are lower indicate slack in that variable. For instance DMU 13 has a DEA score of $84 \%$ but closer inspection shows that for PGR the efficiency is $68 \%$, only $58 \%$ for PGT, and $78 \%$ for UG. Consequently it comes as no surprise to find that its new ranking is lower, at 21, falling from 14 under DEA. Obviously when some units fall in the rankings the ones whose slacks are smaller will tend to rise. For example DMU 17 only had slack in one of the four outputs (PGR); as a result its rank rose from 12 th to 4 th.

Note that the frontier models we have discussed have only one term per variable. If we take the initial linear model and add a quadratic term for each of the two inputs we find the 
correlation rises to 0.990 . To ensure that the contribution from each input is positive one includes constraints of the form $a \mathrm{x}+a^{\prime} \mathrm{x}^{2} \geq 0$ where $a$ and $a^{\prime}$ are free in sign.

For inefficient units standard DEA models provide target values for individual outputs or inputs based on a radially projected point on the frontier followed by the addition of any slack. With the approach presented here there is no slack to account for and we deduce radial target values by simply dividing the current output level by the efficiency score. Of course efficiency is attained at any point on the frontier, the radial target is merely the special case where all outputs are expanded by the same factor. Whilst standard DEA can provide the equation of the particular linear facet upon which the radial projection point lies, this does not permit investigating paths to efficiency which require significant changes in input or output mix as these would involve other facets of the frontier. DEA can be adapted to explore the latter by incorporating user preferences by solving further linear programmes ${ }^{20}$. By contrast our frontier model is global and smooth, so we can investigate any input or output mix whatever: one has a simple equation relating the inputs to the outputs for efficiency to be achieved, e.g. for our second attempted model, substitution of (1) into (2) shows it to be:

$$
\mathrm{RSCH}+23.63 \mathrm{UG}+80.3 \mathrm{PGT}+70.4 \mathrm{PGR}=7204(\ln \mathrm{GEN}+0.1036 \ln \mathrm{EQ})-42973
$$

\section{Conclusion}

In the past there has been little effort to cross-fertilise parametric and non-parametric approaches for efficiency estimation. We have shown that by doing so we can not only maintain the best qualities of each, but also avoid many of their respective disadvantages. In particular we avoid assuming a particular distribution of efficiencies as is done in stochastic frontier analysis and, by separating the process of identifying efficient units from the modelling of the frontier we are able to build a model of the frontier which is not influenced by the presence of inefficient units. The key new feature in our hybrid procedure is that of maximum correlation modelling (MCM, constrained canonical correlation analysis). This powerful new tool was used to fit a surface over the efficient production units and thereby create a benchmark frontier from which the efficiency of other units could be assessed. This technique naturally extends regression-based approaches for efficiency assessment to the case of multiple outputs and multiple inputs in a single model. Just as regression fits a function or index of the independent variables (inputs) so as to maximise the correlation with the dependent variable (output), so MCM fits such a function to maximise correlation with a function or index of multiple outputs. Furthermore, the ability to include constraints permits us to construct models which satisfy requirements from theory or for meaningfulness. Other advantages of our 
approach include the fact that the scores give a full reflection of inefficiency (i.e. slacks are avoided), and that this now implies that ranking of the production units based on their scores is less unreasonable than under DEA because there is now a single formula to calculate the score.

Only a small part of the DEA literature is devoted to performance management, a recent example being Storbeck and Waring's ${ }^{21}$ work on performance-based budgeting to link resource allocation decisions to measured results. In our approach managers can easily investigate the effect of adjusting input and output levels on their performance because there is now a simple formula for calculating efficiency. (Of course such an estimate will be based on the existing data and does not involve any predictions regarding future shifts in the frontier.) Furthermore the modelling of the frontier is based on fitting a smooth continuous function to all the best-practice candidate units simultaneously and so, unlike DEA, does not allow a single maverick unit to adopt extreme weights to achieve the appearance of being efficient. Such mavericks will tend to find that the model assigns them a less generous score than under DEA alone.

It is worth emphasising that maximum correlation modelling is a technique of much broader application since it is effectively a generalisation of regression to the case of multiple dependent variables in a single equation ${ }^{22}$. As it can be implemented on a spreadsheet package (using the Solver tool) there is wide scope for its immediate employment.

Acknowledgements - I wish to express my gratitude to the referees for suggestions which have helped improve the paper.

\section{Appendix}

We here set out some mathematical details associated with finding the coefficients $(\mathbf{a}, \mathbf{b})$ in the maximum correlation model for given data matrices $\mathbf{X}$ and $\mathbf{Y}$ (not to be confused with the vectors of weighted inputs and outputs $\mathrm{X}$ and $\mathrm{Y}$, also known as canonical variates). $\mathbf{X}^{\prime}$ denotes the transpose of $\mathbf{X}$.

The optimisation problem is:

$$
\begin{aligned}
& \max r=\operatorname{Cov}(X, Y) /[\operatorname{Var}(X) \operatorname{Var}(Y)]^{1 / 2}=\mathbf{a}^{\prime} \mathbf{X}^{\prime} \mathbf{Y} \mathbf{b} /\left[\left(\mathbf{a}^{\prime} \mathbf{X}^{\prime} \mathbf{X} \mathbf{a}\right)\left(\mathbf{b}^{\prime} \mathbf{Y}^{\prime} \mathbf{Y} \mathbf{b}\right)\right]^{1 / 2} \\
& \text { subject to any constraints deemed necessary, for example } \mathbf{a}, \mathbf{b} \geq 0
\end{aligned}
$$

Note that one will always need some form of additional constraint to uniquely identify the coefficients since multiplying $\mathbf{a}$ or $\mathbf{b}$ by a constant leaves the correlation unchanged. In the paper we achieved this by setting one each of the input and output coefficients to unity. As already mentioned in the paper we used a general-purpose non-linear solver for the calculations and have not gone into the technicalities of the solution algorithm. We have since discovered that work has been done on this aspect by Hayashi and Panter ${ }^{23}$ who use Kuhn Tucker Lagrangian theory to derive necessary conditions for optimality. For the special case of linear equality constraints see Yanai and Takane ${ }^{24}$. 


\section{References}

1. Greene WH (1993). The econometric approach to efficiency analysis. In: H.O. Fried, C.A. Knox Lovell and S.S. Schmidt (eds.), The Measurement of Productive Efficiency, Oxford University Press, NY and Oxford, pp 68-119.

2. Simar L (1992). Estimating efficiencies from frontier models with panel data. $J$ Productivity Analysis 3: 171-203.

3. Bessent A, Bessent W, Elam J and Clark T. (1988). Efficiency frontier determination by constrained facet analysis. Ops Res 36: 785-796.

4. Torgersen AM, Forsund FR, Kittelsen SAC (1996). Slack-adjusted efficiency measures and ranking of efficient units. J Productivity Analysis 4: 379-398.

5. Lovell CAK and Eeckaut PV (1993). Frontier Tales: DEA and FDH. In: Diewert, W.E. et al.(Eds.) Mathematical Modelling in Economics, Springer: Berlin, pp. 446-457.

6. Hartman TE, and Storbeck JE (1998). Managerial endorsement of data envelopment analysis. In: Neely $\mathrm{AD}$ and Waggoner DB (eds). Performance Measurement - Theory and Practice. Centre for Business Performance, Judge Inst., Cambridge University.

7. Thiry B and Tulkens H (1992). Allowing for inefficiency in parametric estimation of production functions for urban transit firms. J Productivity Analysis 3: 45-65.

8. Sengupta JK (1989). Data envelopment with Maximum Correlation. Int J of Systems Science 20: 2085-2093.

9. Friedman L and Sinuany-Stern Z (1997). Scaling units via the canonical correlation analysis in the DEA context. Eur J Opl Res 100: 629-637.

10. Arnold VL, Bardhan IR, Cooper WW and Kumbhakar SC (1996). DEA and Statistical Regressions for Efficiency Evaluation and Estimation. Annals Ops Res 66: $255-277$.

11. Manly BFJ (1994). Multivariate Statistical Methods: A Primer (2nd edn). Chapman and Hall: London.

12. Beasley JE (1990). Comparing university departments. Omega 18: 171-183.

13. Beasley JE (1995). Determining teaching and research efficiencies. J Opl Res Soc 46: 441-452.

14. Gittins R (1985). Canonical Analysis: A Review with Applications is Ecology Springer-Verlag: Berlin. 
15. Tabachnick BG and Fidell LS (1983). Using Multivariate Statistics Harper and Row: New York

16. Charnes A, Cooper WW, and Huang ZM (1990). Polyhedral cone-ratio DEA with an illustrative application to commercial banks. J Econometrics 46: 73-91.

17. Allen R, Athanassopoulos A, Dyson RG, Thanassoulis E (1997). Weights restrictions and value judgements in data envelopment analysis. Annals Ops Res 73: $13-34$.

18. Dyson RG, and Thanassoulis E (1988). Reducing weight flexibility in data envelopment analysis. J Opl Res Soc 39(6): 563-576.

19. Doyle J and Green R (1994). Efficiency and cross-efficiency in DEA: Derivations, meanings and uses. J Opl Res Soc 45(3):567-578.

20. Thanassoulis E. and Dyson R.G. (1992). Estimating preferred target input-output levels using DEA. Eur J Opl Res 56: 80-97.

21. Storbeck JE and Waring CG (2000). Model support for performance-based budgeting. In: Neely A (ed). Performance Measurement - Past, Present and Future. Centre for Business Performance, Cranfield School of Management, Cranfield University.

22. Tofallis C (1999). Model building with multiple dependent variables and constraints. The Statistician 48(3): 371-378.

23. Hayashi K and Panter AT (1994). Canonical correlation analysis with both equality and inequality constraints. Paper presented at the Joint Statistical Meetings of the American Statistical Association.

24. Yanai H and Takane Y (1992). Canonical correlation analysis with linear constraints. Linear Algebra and its Applications 176 75-89. 


\begin{tabular}{|c|c|c|c|c|c|c|}
\hline \multirow{2}{*}{ Dept. } & \multicolumn{4}{|c|}{ DEA (actual output/target output) \% } & DEA & New \\
& PGR & PGT & \multicolumn{1}{c|}{ RSCH } & UG & Rank & Rank \\
\hline 13 & 68 & 58 & 84 & 78 & 14 & 21 \\
\hline 17 & 78 & 87 & 87 & 87 & 12 & 4 \\
\hline
\end{tabular}

Table 1 The (radial) DEA score is the largest of the ratios shown for the various outputs. Hence using this for ranking purposes hides inefficiencies due to slacks in some outputs. Only if the DEA score for each output were the same would there be no slack. (N.b. these rankings are based on the inefficient units alone.) 
Figure 1 Illustration of DEA (radial) measurement and the problem of slack.

Table 1 The (radial) DEA score is the largest of the values for the various outputs. Hence using this for ranking purposes hides inefficiencies due to slacks in some outputs. Only if the DEA score for each output were the same would there be no slack. (N.b. these rankings are based on the inefficient units alone.) 
Figure 1

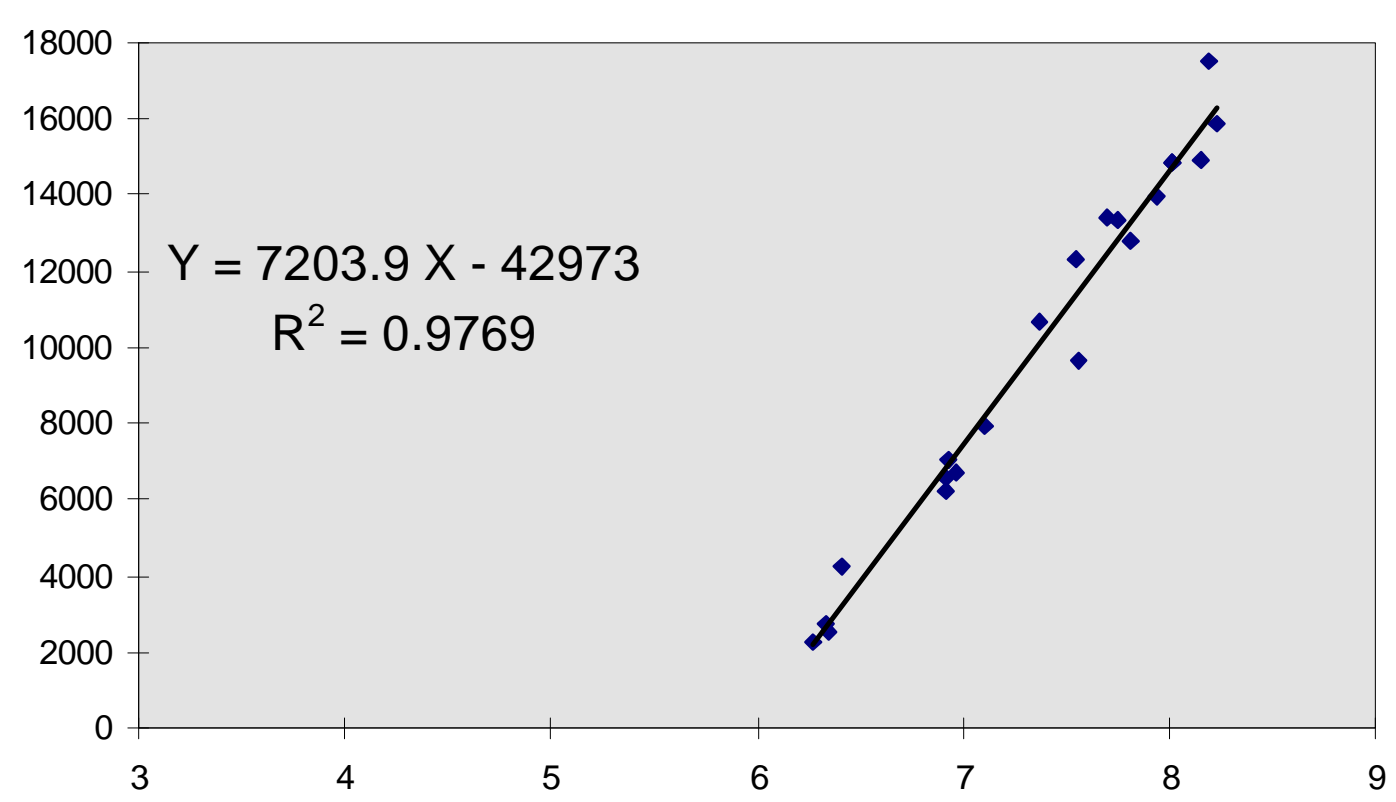


Figure 1: Illustration of radial DEA measurement and the problem of slack.

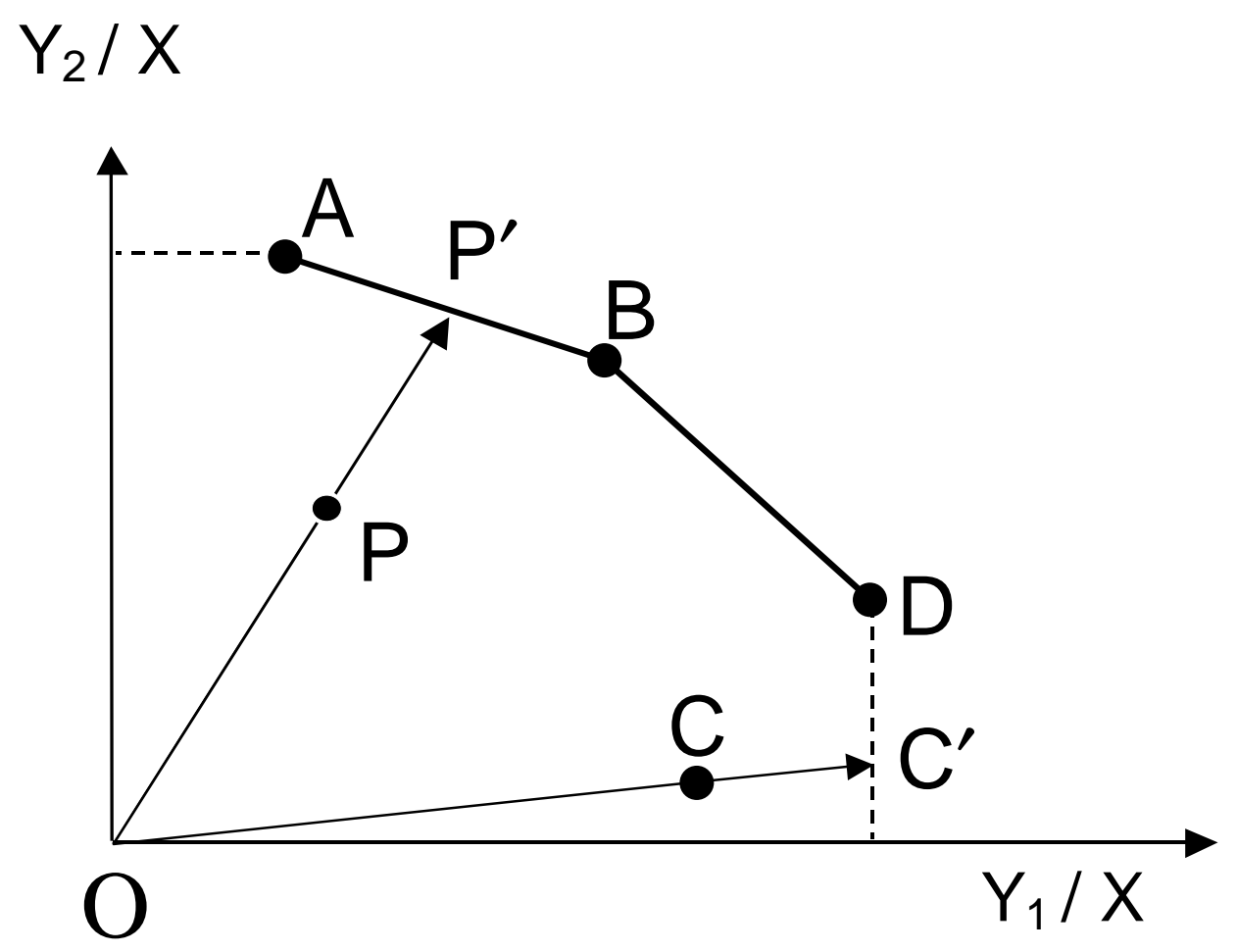

